\title{
Lack of advantages of slit mesh placement during laparoscopic transabdominal preperitoneal inguinal hernia repair (TAPP): a single centre, case matched study
}

\author{
Umberto Bracale $^{2^{*}}$ D, Jacopo Andreuccetti ${ }^{1}$, Maurizio Sodo ${ }^{1}$, Giovanni Merola ${ }^{1,2}$ and Giusto Pignata ${ }^{1}$
}

\begin{abstract}
Background: During laparoscopic trans-abdominal pre-peritoneal hernia repair (TAPP) the positioning of the mesh around the spermatic cord could provide an additional anchoring point and ensure better defect closure, thereby preventing mesh movement and recurrence. The primary aim of our retrospective study was to determine if, during a TAPP procedure, an advantageous difference for mesh placement exists between the slit and the non-slit techniques in terms of recurrence rate. Secondary aims were intra and post-operative complications and the time required to return to normal activity.

Methods: From January 2010 to December 2015, data from patients who had undergone TAPPs at our Institution were prospectively collected. We performed a retrospective case control matched study of two homogenous (BMI, Age, type of hernia) groups of 100 patients who underwent respectively TAPP with no slit mesh placement (Group NS) and slit mesh placement (Group S). Statistical analysis was carried out using a SPSS 20. To compare continuous variables, an independent sample T-test was performed. A Chi-square test was employed for categorical data.

Results: No differences were found between the slit and non-slit groups in terms of biometric features and intra and post-operative outcomes were found to be similar in both groups as well. In particular, at mean follow-up of $57.34 \pm 10.56$ months for Group NS and $55.66 \pm 8.61$ months for Group S months only one recurrence per group was found.

Conclusion: Our study failed to prove a superiority of the slit mesh technique over the no-slit mesh technique during TAPP. However, in light of its not being a randomized study, a subsequent, well-designed RCT would be desirable in order to better determine if the Slit mesh technique could prove to be advantageous enough to justify its routine use during the TAPP procedure.
\end{abstract}

Keywords: Laparoscopy, No slit vs slit Mesh, Inguinal hernia repair, TAPP, Laparoscopic hernia repair

\section{Background}

Inguinal hernia repair (IHR) is one of the most common procedures in general surgery worldwide and can be performed with both an open or laparoscopic approach (LA). Every year more than 800,000 IHRs are performed in the United States alone [1]. LA has the added advantage of combining mesh inguinal hernia repair with a

\footnotetext{
* Correspondence: umbertobracale@gmail.com

${ }^{2}$ Department of Surgical Specialities and nephrology, University Federico II Naples, Via Pansini 5, 80131 Naples, Italy

Full list of author information is available at the end of the article
}

minimally invasive technique thereby proving less painful and enabling a faster return to work and daily activities as well as having better aesthetic outcome. As far as we are aware, no differences between the Transabdominal preperitoneal hernia repair (TAPP) and the Totally extra-peritoneal hernia repair (TEP) approach has ever been reported in published literature [2].

The International Endohernia Society (IEHS) guidelines indicate to use a macroporose lightweight mesh sized up to $10 x 15 \mathrm{~cm}$, non-fixed or fixed with fibrin glue. Some controversy, due to the lack of strong evidences,

(c) The Author(s). 2018 Open Access This article is distributed under the terms of the Creative Commons Attribution 4.0 International License (http://creativecommons.org/licenses/by/4.0/), which permits unrestricted use, distribution, and reproduction in any medium, provided you give appropriate credit to the original author(s) and the source, provide a link to the Creative Commons license, and indicate if changes were made. The Creative Commons Public Domain Dedication waiver (http://creativecommons.org/publicdomain/zero/1.0/) applies to the data made available in this article, unless otherwise stated. 
arises around the slitting or non-slitting of the mesh in order to wrap it around the spermatic cord during laparoscopic inguinal hernia repair (LHR) $[3,4]$. Some surgeons presume that a slit mesh wrapped around the cord, thereby fashioning a new internal ring, could prevent a hernia recurrence which, however, could cause circumferential scarring with subsequent postoperative pain. No evidence exists on spermatic cord injury nor a reduction in the recurrence rate due to the slit procedure has been published [5-11]. To date, only nine studies have compared the results of slit vs. no-slit mesh techniques, six during a TEP $[5,6,12-15]$ and three others during a TAPP $[10,11,16]$.

The primary aim of our case matched retrospective study was to determine if, during a TAPP procedure, an advantageous difference for mesh placement exists between the slit and the non-slit techniques in terms of recurrence rate, secondary aims were intra and post-operative complications and the time required to return to normal activity.

\section{Methods}

This retrospective analysis of data was approved by the internal ethical commitee (Protocol $n^{\circ} 71 / 2018$ of San Camillo Hospital of Trento ethical committee), we performed a retrospective analysis of data from patients who had undergone a TAPP in the Department of General and Mini-invasive surgery of San Camillo Hospital in Trento from January 2010 to December 2015. During this period a total of 516 patients underwent TAPP, of these 297 with no slit mesh placement and 219 with slit mesh placement. In order to avoid bias only male patients were included in the analysis. After female patients exclusion there were 267 patients with no slit mesh placement and 199 with slit mesh placement. All patient before surgery signed an informed consent for the surgical procedure and data collection.

We performed a retrospective case control matched study of two homogenous (BMI, Age, type of hernia) groups of 100 patients who underwent respectively TAPP with no slit mesh placement (Group NS) and slit mesh placement (Group S).

Indications for a LHR were bilateral inguinal hernia, recurrent inguinal hernia and monolateral inguinal hernia in young or sportsmen patients.

Contraindications included patient preference for open repair, ASA IV, and previous prostatectomy.

All procedures were performed by three different surgeons who had completed their learning curve and each of them had done at least 150 laparoscopic inguinal hernia repairs before.

Data collected included: gender, age, American Society of Anaesthesiologists risk class (ASA), Body Mass Index (BMI), operative time (OT), type of hernia according to
IEHS classification [17], conversion rate, intra and post-operative complications such as bleeding, seroma, wound infection, numbness (evaluated at 1 week, at 1 month, 6 months and permanent), chronic pain, recurrence rate, hospital stay and time to return to normal activity. Chronic pain is defined as pain lasting 6 months or more [18-20]. Follow-up with clinical examination was conducted at 7 days, 1 month and every year from surgery.

\section{Statistical analysis}

Statistical analysis was performed using IBM SPSS Statistics 23. The case matching was assessed only for biometrical features. Continuous data were expressed as mean \pm Standard deviation (SD). To compare continuous variables, an independent sample T-test was performed. The Chi-square test was employed to analyse categorical data. All results are presented as 2-tailed values with statistical significance if $p$ values $<0.05$. Logistic regression was performed to assess if there were any correlation between complications and other factors.

\section{Perioperative management}

Before surgery all patient had undergone a physical examination and a routine blood test only. In accordance with IEHS guidelines, antibiotics and thromboembolic prophylaxis were administered only in select cases [3]. An intraoperatively nasogastric tube and urinary catheter were placed and removed at the end of each procedure. Pain management was achieved by non-steroidal anti-inflammatory drugs (NSAIDs) which were administered when required. All patients resumed drinking in the evening following surgery. Most were discharged on the first postoperative day.

\section{Surgical technique}

The surgical technique for laparoscopic inguinal hernia repair is the same described in our previous paper [21]. Under general anaesthesia the patient was placed in a Trendelenburg position $\left(30^{\circ}\right)$ with arms and legs adduced. Pneumoperitoneum was established with the Veress technique [22]. The first $10 \mathrm{~mm}$ trocar was placed in the umbilicus, and two other $5 \mathrm{~mm}$ ports were placed bilaterally in the midclavicular line $1 \mathrm{~cm}$ below the umbilical line (Fig. 1). After identification of the anatomical landmarks (Epigastric Vessels, Spermatic vessels, vas deferens or round ligament, urachus, iliac vessels and bladder), the preperitoneal space was opened incising the peritoneum transversely from the region of the umbilical artery laterally to the hernia defect. The dissection was carried out into the Retzius and Bogros (retroinguinal) spaces. The anatomical landmarks (epigastric vessels, Cooper and Gimbernat ligaments, the corona mortis and external iliac vessels) were then 


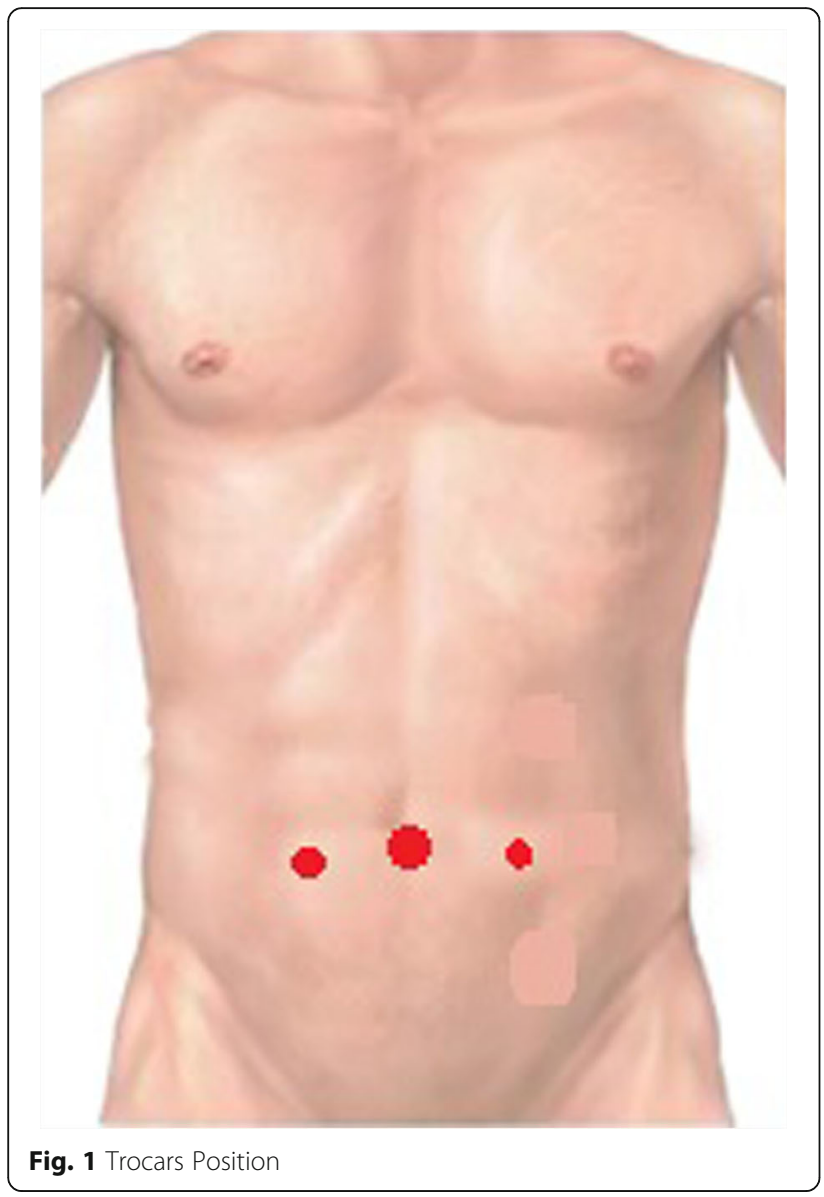

identified and well exposed. The sac dissection was performed carefully safeguarding the spermatic fascia and protecting the fragile parietal structures.

A $10 \times 15 \mathrm{~cm}$ polyester mesh was placed directly over the cord structures (Group NS) (Fig. 2), or "key-holed", after engraving vertically the mesh in the upper part, to accommodate the cord (Group S) (Fig. 3). The mesh was securely fixed with $2 \mathrm{ml}$ of fibrine glue (Tisseel/Tissucol,

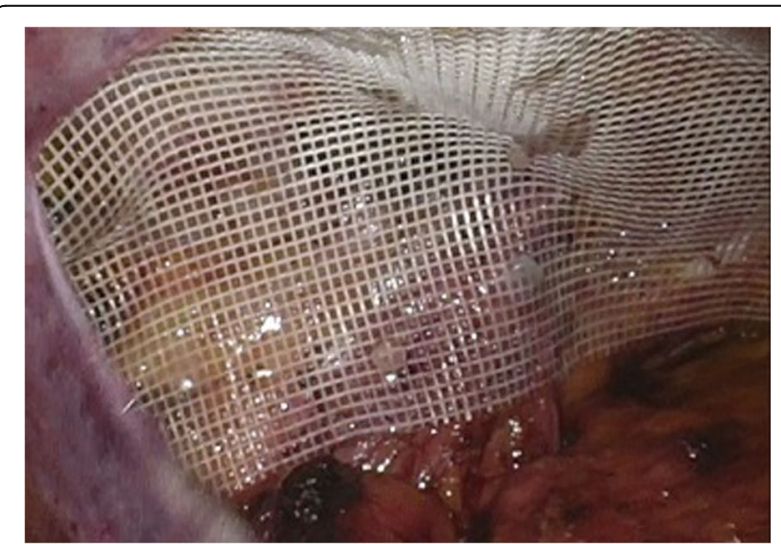

Fig. 2 Non-Slit Mesh Placement

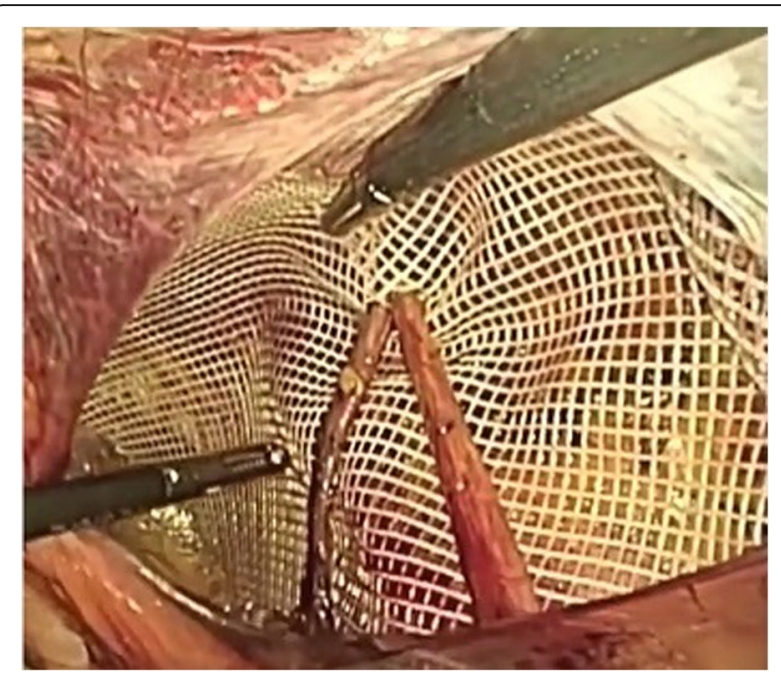

Fig. 3 Slit Mesh Placement

Baxter Healthcare, Deerfield, IL, USA) in order to avoid vessel injury or nerve entrapment, in accordance with IEHS guidelines [4]. The peritoneum was closed with a running suture in V-Loc 3/0, and trocars were removed under direct visualization. The fascial defect of $10 \mathrm{~mm}$ port was then closed under direct visualization.

\section{Results}

No differences in biometric features existed between the two groups as reported in Table 1. As well, no variations in the distribution of unilateral and bilateral hernia between the two groups were noted such as that for the distribution of recurrent and primitive hernia. Intra and post-operative outcomes are reported in Table 2. Global operative time was comparable between the two groups. No statistical differences in OT for bilateral inguinal hernia was noted in either of the two groups (Group NS $85 \pm 23.21 \mathrm{~min}$; Group S $91.92 \pm 22.75 \mathrm{~min}, p=0.097$ ) such as that noted for OT in unilateral inguinal hernia (Groups NS 77.2 \pm 28.28 min; Group S $72.28 \pm$ 17.59 min, $p=0.45)$. No conversions nor intraoperative complications had occurred in either group. Eleven and nine early, post-operative complications occurred in Group NS and Group S respectively. Seroma was the most frequent complication and only one bleeding and wound infection were found per group. The two bleeding reported (one per group) are of the navel as well as the two wound infections (one per group).

No statistical difference was observed in length of hospital stay between the two groups and return to daily activity was comparable within both groups. No statistically significant difference occurred in recurrence, numbness and chronic pain between the groups. The median follow-up was $57.34 \pm 10.56$ months (Range 3066 months) for Group NS and $55.66 \pm 8.61$ months 
Table 1 Biometric Features

\begin{tabular}{|c|c|c|c|}
\hline & Group NS 100pts & Group S 100pts & $\mathrm{P}$ \\
\hline Age (Mean \pm SD) & $56.3 \pm 12.01$ & $58.17 \pm 12.2$ & 0.295 \\
\hline BMI (Mean \pm SD) & $25.53 \pm 3.52$ & $25.47 \pm 3.77$ & 0.912 \\
\hline Type of Hernia ( $N^{\circ}$ Unilateral/Bilateral) & $30 / 70$ & $27 / 73$ & 0.428 \\
\hline Type of Hernia ( $N^{\circ}$ Primitive/Recurrent) & $160 / 10$ & $165 / 8$ & \\
\hline \multicolumn{4}{|c|}{ Type of Hernia according to EHS classification } \\
\hline PLOMIFO & 9 & 7 & \\
\hline PLOM2FO & 22 & 22 & \\
\hline PLOM3FO & 12 & 10 & \\
\hline PL1M2FO & 17 & 16 & \\
\hline PL2M2FO & 9 & 11 & \\
\hline PL3M2FO & 2 & 3 & \\
\hline PL2M1FO & 2 & 1 & \\
\hline PL1M3FO & 1 & 2 & \\
\hline PLIMOFO & 29 & 31 & \\
\hline PL2MOFO & 36 & 38 & \\
\hline PL3MOFO & 8 & 10 & \\
\hline PLOMOF1 & 3 & 4 & \\
\hline PL2MOF1 & 4 & 5 & \\
\hline PL2MOF2 & 2 & 2 & \\
\hline PLOM2F1 & 4 & 3 & \\
\hline RL2MOFO & 3 & 1 & \\
\hline RLIMIFO & 1 & 1 & \\
\hline RLOM1FO & 1 & 2 & \\
\hline RLOM2FO & 2 & 1 & \\
\hline RLOM3FO & 2 & 2 & \\
\hline RLOMOF1 & 1 & 1 & \\
\hline \multicolumn{4}{|l|}{ ASA Classification } \\
\hline । & 34 & 40 & \\
\hline$\|$ & 48 & 45 & \\
\hline III & 18 & 15 & \\
\hline
\end{tabular}

(Range 24-64 months) for Group S (Table 2). We lost at follow-up 3 patients (3\%) in group NS and 2 patients (2\%) in group S.

\section{Discussion}

Since 1990, when the first LHR repair was described, LA for treatment of groin hernia has been widely practiced [5]. TAPP and TEP techniques guarantee the same results as open inguinal hernia repair in terms of recurrence, which add to the advantages of LA over conventional surgery, such as less pain, faster return to daily activities and better aesthetic outcome. Some controversies arise around the slitting or not of the mesh employed in order to place it around the spermatic cord. Some surgeons presume that a slit mesh closed around the cord making a new internal ring, could prevent a recurrence albeit resulting in circumferential scarring with subsequent postoperative pain.

After a literature review, we found nine articles on the slit vs. non-slit topic $[5,6,10-16]$ (Table 3). Leibl et Al. [10], in their three-arm randomized trial, reported no difference in the recurrence rate, OT, postoperative complications and pain killer assumption between their group A (employing a slit mesh) and the other two study groups (No slit mesh fixed with staples in group B or suture in group $\mathrm{C}$ ). Their results found only one recurrence in Group C. Leibl et $\mathrm{Al}$ [23], also published a previous paper on the causes of inguinal hernia recurrence after TAPP, identifying incision of the mesh as a "second cause of recurrence". However, in the above-mentioned 
Table 2 Intra and Post-operative outcomes

\begin{tabular}{|c|c|c|c|}
\hline & Group NS 100pts & Group S 100pts & $P$ \\
\hline Conversion Rate $\mathrm{n}^{\circ}$ & 0 & 0 & 1 \\
\hline Global Operative Time (Mean \pm SD) in min & $83.04 \pm 24.99$ & $87.01 \pm 23.13$ & 0.245 \\
\hline Operative Time in bilateral hernia (Mean \pm SD) in min & $85 \pm 23.21$ & $91.92 \pm 22.75$ & 0.097 \\
\hline Operative Time in unilateral hernia (Mean \pm SD) in min. & $77.2 \pm 28.28$ & $72.28 \pm 17.59$ & 0.45 \\
\hline Intraoperative complications $n^{\circ}$ & 0 & 0 & 1 \\
\hline \multicolumn{4}{|l|}{ Post-Operative Complication } \\
\hline Seroma (\%) & 9 & 7 & 0.602 \\
\hline Bleeding (\%) & 1 & 1 & 1 \\
\hline Wound Infection (\%) & 1 & 1 & 1 \\
\hline Hospital Stay (Mean \pm SD) in days & $1.04 \pm 0.19$ & $1.06 \pm 0.23$ & 0.519 \\
\hline Return to work in days (Mean $\pm S D$ ) & $15.88 \pm 1.94$ & $15.77 \pm 0.23$ & 0.718 \\
\hline \multicolumn{4}{|l|}{ Numbness } \\
\hline 1 week (\%) & 8 & 12 & 0.816 \\
\hline 1 month (\%) & 2 & 4 & 0.407 \\
\hline 6 months (\%) & 0 & 0 & 1 \\
\hline Permanent & 0 & 0 & 1 \\
\hline Chronic Pain (\%) & 0 & 1 & 0.316 \\
\hline Recurrence (\%) & 1 & 1 & 1 \\
\hline
\end{tabular}

trial, they concluded that mesh fashioning alternatives did not result in any differences in postoperative complaints or complications.

Leibl's results are consistent with Celik et al.'s [5] previous study whereby they confirm that LHR has no negative effect on testicular blood flow and volume, nor on recurrence, in both Slit or No-Slit groups. Korman et al. [24], in their study on the fashioning of differently cut meshes, did not find any resulting differences in terms of recurrence and chronic pain.

Domniz et al. [6] evaluated the efficacy of slit mesh during TEP, reporting a statistically significant recurrence rate in favour of the slit group. They argued that their results, as a consequence of the wrapping of the mesh below the spermatic cord which provided an additional anchoring point and better defect closure, preventing mesh movement and recurrence. This hypothetical advantage could prove to be more effective in the presence of large indirect hernia. Domnniz's group did find, however, that the slit mesh surgery took about 8 min longer than the non-slit procedure, implying that the Slit procedure could be a more challenging technique.

About the relatively high seroma rate found in both groups of our study, it could be explained for two reasons, in accordance with those reported in a recent study [25]. In the study period, we didn't use any technical tricks to prevent the seroma development, as well as the ligation of Transversalis Fascia trough the Röder loop or the fixation of the Transversalis Fascia to the

Table 3 Previous paspers about Slit VS non-Slit mesh placement technique

\begin{tabular}{lllll}
\hline Author & Technique & Use of Slit & Number of patients in slit group & Recurrence (\%) \\
\hline Velasco & TAPP & Horizzontal slit & 25 & 6 \\
Leibl 2002 & TAPP & Vertical slit & 124 & 0 \\
Leibl 1998 & TAPP & Vertical slit & 2700 & 1.03 \\
McKernan & TEP & Vertical slit & 34 & $?$ \\
Phillips & TEP & Oblique slit & 172 & 0 \\
Chia & TEP & Oblique slit & 54 & 1.6 \\
Cristaudo & TEP & NR & 14 & NR \\
Celik & TEP & Vertical slit & 20 & 0 \\
Domniz & TEP & Vertical slit & 87 & 0.6
\end{tabular}

Trans abdominal preperitoneal hernia repair (TAPP); Totally extraperitoneal hernia repair (TEP), Not Reported (NR) 
Cooper Ligament through a suture or through a not-absorbable tack. Secondarily, it is not negligible the rate of Large defects (L3 or M3) collected in our series. As reported by Köckerling et Al., a large hernia defect has a significantly higher risk of seroma formation. Finally, in all cases we had used the fibrin glue as fixation method, which presents a higher risk for seroma development in TAPP inguinal hernia repair [25].

Although the aim of our study did not commit the testis vascularization, no differences in inguinal or scrotal long-term numbness, nor testicular atrophy between the two groups were noted. Only a greater numbness in Group S (Group NS: 8; Group S: 17, p 0.054) during the first post-operative week was found. These results may be due to the greater surgical stress of the genital branch of the genito-femoral nerve during preparation of the spermatic cord structures yet the degree of numbness noted at 1 month was similar amongst the two groups. Similarly, no statistically significant differences in recurrence and chronic pain between the two groups was noted.

Although no consensus was found in the literature, IEHS recommends (Grade B) not cutting the mesh in order to allow for passage of the spermatic cord structures. This is because this technique does not necessarily reduce the recurrence rate and may cause injury to the funicle structures [4]. This recommendation is not supported by strong evidence concerning suspected risk of funicle structure injury nor postoperative testicular atrophy; however, it could be a consequence of a lack of advantages in terms of recurrence prevention.

Our results are consistent with IEHS' recommendation even if we did not find the use of a slit mesh less safe, more risky or more time consuming then the use of a no slit mesh. Also, the recurrence rate in the subgroup of lateral defects in which the indication of a slit mesh would be more indicated, was not found to be different to that of the other subgroups (medial or femoral defects).

\section{Conclusion}

In conclusion, our study, did not demonstrate the superiority of the slit mesh technique over the non-slit mesh technique in a TAPP. For this reason, a well-designed RCT is desirable in order to clarify if the Slit mesh technique has any advantages over the non-Slit technique to justify its routine employment in transabdominal inguinal hernia repair.

\footnotetext{
Abbreviations

ASA: American Society of Anaesthesiologists risk class; BMI: Body Mass Index; IEHS: International endo hernia society; IHR: Inguinal hernia repair; LA: Laparoscopic approach; LHR: Laparoscopic hernia repair; NS: Non- slit; OT: Operative time; RCT: Randomized controlled trial; S: Slit; SD: Standard deviation; TAPP: Trans abdominal pre peritoneal hernia repair; TEP: Totally extra peritoneal hernia repair
}

\section{Acknowledgements}

We thanks Juliet Ippolito, B.A. Vassar College, MPhil University of Dundee for English language editing.

\section{Availability of data and materials}

The datasets used during the current study available from the corresponding author on reasonable request.

\section{Authors' contributions}

UB article writing and review. JA data collection and article writing. MS article reviewing. GM analysed and interpreted data, article writing. GP article writing and review. All authors read and approved the final manuscript.

Ethics approval and consent to participate

This retrospective analysis of data was approved by the internal ethical committee (Protocol n ${ }^{\circ} 71 / 2018$ of San Camillo Hospital of Trento ethical committee)

\section{Consent for publication}

Before surgery, each patient signed a written consent for the surgical procedure and data collection. In any case, the images and data shown make it impossible to identify any patient in any way.

\section{Competing interests}

All of the authors have no potential conflicts of interest to disclose with respect to the research, authorship, and publication of this article. All authors received no financial support for the research, authorship, and publication of this article.

\section{Publisher's Note}

Springer Nature remains neutral with regard to jurisdictional claims in published maps and institutional affiliations.

\section{Author details}

${ }^{1}$ Department of General and Mini-invasive Surgery, San Camillo Hospital of Trento, Via Giovanelli 19, 38121 Trento, Italy. ${ }^{2}$ Department of Surgical Specialities and nephrology, University Federico II Naples, Via Pansini 5, 80131 Naples, Italy.

Received: 3 January 2018 Accepted: 12 September 2018

Published online: 20 September 2018

\section{References}

1. Bracale U, Rovani M, Picardo A, Merola G, Pignata G, Sodo M, Di Salvo E, Ratto EL, Noceti A, Melillo P, et al. Beneficial effects of fibrin glue (Quixil) versus Lichtenstein conventional technique in inguinal hernia repair: a randomized clinical trial. Hernia. 2014;18(2):185-92.

2. Bracale U, Melillo P, Pignata G, Di Salvo E, Rovani M, Merola G, Pecchia L. Which is the best laparoscopic approach for inguinal hernia repair: TEP or TAPP? A systematic review of the literature with a network meta-analysis. Surg Endosc. 2012;26(12):3355-66.

3. Bittner R, Arregui ME, Bisgaard T, Dudai M, Ferzli GS, Fitzgibbons RJ, Fortelny $\mathrm{RH}$, Klinge U, Kockerling F, Kuhry E, et al. Guidelines for laparoscopic (TAPP) and endoscopic (TEP) treatment of inguinal hernia [international Endohernia society (IEHS)]. Surg Endosc. 2011;25(9):2773-843.

4. Bittner $R$, Montgomery MA, Arregui $E$, Bansal V, Bingener J, Bisgaard T, Buhck H, Dudai M, Ferzli GS, Fitzgibbons RJ, et al. Update of guidelines on laparoscopic (TAPP) and endoscopic (TEP) treatment of inguinal hernia (international Endohernia society). Surg Endosc. 2015;29(2):289-321.

5. Celik AS, Memmi N, Celebi F, Guzey D, Celik A, Kaplan R, Oncu M. Impact of slit and nonslit mesh technique on testicular perfusion and volume in the early and late postoperative period of the totally extraperitoneal preperitoneal technique in patients with inguinal hernia. Am J Surg. 2009;198(2):287-91.

6. Domniz N, Perry ZH, Lantsberg L, Avinoach E, Mizrahi S, Kirshtein B. Slit versus non-slit mesh placement in total extraperitoneal inguinal hernia repair. World J Surg. 2011;35(11):2382-6.

7. Koeckerling F, Jacob DA, Lomanto D, Chowbey P, Bittner R. Slit versus nonslit mesh placement in total extraperitoneal inguinal hernia repair. World Surg. 2012;36(6):1445-6 author reply 1447-1448. 
8. Rosin D. Slit versus non-slit mesh placement in total extraperitoneal inguinal hernia repair. World J Surg. 2011;35(11):2387-8.

9. Bittner R, Schmedt CG, Schwarz J, Kraft K, Leibl BJ. Laparoscopic transperitoneal procedure for routine repair of groin hernia. Br J Surg. 2002;89(8):1062-6.

10. Leibl BJ, Kraft B, Redecke JD, Schmedt CG, Ulrich M, Kraft K, Bittner R. Are postoperative complaints and complications influenced by different techniques in fashioning and fixing the mesh in transperitoneal laparoscopic hernioplasty? Results of a prospective randomized trial. World J Surg. 2002;26(12):1481-4.

11. Leibl BJ, Schmedt CG, Schwarz J, Daubler P, Kraft K, Schlossnickel B, Bittner R. A single institution's experience with transperitoneal laparoscopic hernia repair. Am J Surg. 1998;175(6):446-51 discussion 452.

12. Cristaudo A, Nayak A, Martin S, Adib R, Martin I. A prospective randomised trial comparing mesh types and fixation in totally extraperitoneal inguinal hernia repairs. Int J Surg. 2015;17:79-82.

13. McKernan JB, Laws HL. Laparoscopic repair of inguinal hernias using a totally extraperitoneal prosthetic approach. Surg Endosc. 1993;7(1):26-8.

14. Phillips EH, Rosenthal R, Fallas M, Carroll B, Arregui M, Corbitt J, Fitzgibbons R, Seid A, Schultz L, Toy F, et al. Reasons for early recurrence following laparoscopic hernioplasty. Surg Endosc. 1995;9(2):140-4 discussion 144-145.

15. Chia CL, Su J, Hoe Y, Shelat VG, Junnarkar SP, Low J, Woon WW. Outcomes of slit mesh in laparoscopic totally extraperitoneal inguinal hernia repair: does it affect recurrence? Asian J Endosc Surg. 2015;8(4):434-8.

16. Velasco JM, Gelman C, Vallina VL. Preperitoneal bilateral inguinal herniorrhaphy evolution of a technique from conventional to laparoscopic. Surg Endosc. 1996;10(2):122-7.

17. Miserez M, Alexandre JH, Campanelli G, Corcione F, Cuccurullo D, Pascual MH, Hoeferlin A, Kingsnorth AN, Mandala V, Palot JP, et al. The European hernia society groin hernia classification: simple and easy to remember. Hernia. 2007;11(2):113-6.

18. Miserez M, Peeters E, Aufenacker T, Bouillot JL, Campanelli G, Conze J, Fortelny R, Heikkinen T, Jorgensen LN, Kukleta J, et al. Update with level 1 studies of the European hernia society guidelines on the treatment of inguinal hernia in adult patients. Hernia. 2014;18(2):151-63.

19. Nikkolo C, Lepner U. Chronic pain after open inguinal hernia repair. Postgrad Med. 2016;128(1):69-75.

20. Alfieri S, Amid PK, Campanelli G, Izard G, Kehlet H, Wijsmuller AR, Di Miceli D, Doglietto GB. International guidelines for prevention and management of post-operative chronic pain following inguinal hernia surgery. Hernia. 2011;15(3):239-49.

21. Bracale U, Sciuto A, Andreuccetti J, Merola G, Pecchia L, Melillo P, Pignata G. Laparoscopic recurrent inguinal hernia repair during the learning curve: it can be done? Ann Ital Chir. 2017:88(1):4.

22. Bracale U, Azioni G, Rosati M, Barone M, Pignata G. Deep pelvic endometriosis (Adamyan IV stage): multidisciplinary laparoscopic treatments. Acta Chir lugosl. 2009;56(1):41-6.

23. Leibl BJ, Schmedt CG, Kraft K, Ulrich M, Bittner R. Recurrence after endoscopic transperitoneal hernia repair (TAPP): causes, reparative techniques, and results of the reoperation. J Am Coll Surg. 2000;190(6):651-5.

24. Korman JE, Hiatt JR, Feldmar D, Phillips EH. Mesh configurations in laparoscopic extraperitoneal herniorrhaphy. A comparison of techniques. Surg Endosc. 1997;11(11):1102-5.

25. Kockerling F, Bittner R, Adolf D, Fortelny R, Niebuhr H, Mayer F, Schug-Pass C. Seroma following transabdominal preperitoneal patch plasty (TAPP): incidence, risk factors, and preventive measures. Surg Endosc. 2018;32(5): 2222-31.

Ready to submit your research? Choose BMC and benefit from:
- fast, convenient online submission
- thorough peer review by experienced researchers in your field
- rapid publication on acceptance
- support for research data, including large and complex data types
- gold Open Access which fosters wider collaboration and increased citations
- maximum visibility for your research: over 100M website views per year
At BMC, research is always in progress.
Learn more biomedcentral.com/submissions

